\title{
Very long baseline interferometry: accuracy limits and relativistic tests
}

\author{
Robert Heinkelmann ${ }^{1}$ and Harald Schuh ${ }^{2}$ \\ ${ }^{1}$ Deutsches Geodätisches Forschungsinstitut DGFI, \\ Alfons-Goppel-Str. 11, 80539 München, Germany \\ email: heinkelmann@dgfi.badw.de \\ ${ }^{2}$ Institute of Geodesy and Geophysics, Vienna University of Technology, \\ Gusshausstr. 27-29, 1040 Wien, Austria \\ email: harald.schuh@tuwien.ac.at
}

\begin{abstract}
We present a review on relativistic effects and best estimates of the relativistic PPN parameter $\gamma$ obtained by analysis of data from the International VLBI Service for Geodesy and Astrometry (IVS). Relativistic implications are also considered in view of the upcoming new generation VLBI System: VLBI2010.
\end{abstract}

Keywords. VLBI, PPN parameter $\gamma$, VLBI2010

\section{Introduction}

Since the foundation of relativity by Einstein (1908) many groups have sought to verify this remarkable theory. Einstein (1911) himself was the first proposing an experimental test of the deflection of light by the Sun by observing star light very close to the Sun during a total solar eclipse. Such tests were actually carried out during almost all solar eclipses from 1919 on, e.g. by the Texas Mauritanian Eclipse Team (1976) and led to first experimental proofs of Einstein's theory within about $\pm 20 \%$ uncertainty. Some years earlier, Very Long Baseline Interferometry (VLBI) came up and it was Shapiro (1967), who had the idea to utilize this new technique for the detection of light deflection. Seielstad et al. (1970) were among the first to realize radio interferometry for gravitational deflection in practice.

\section{Determination of the PPN parameter $\gamma$ by VLBI}

The relativity in this context is usually described by the parameterized post-Newtonian (PPN) formalism (cf. Soffel, 1989), which defines a number of PPN parameters numerically expressing certain interactions between time, space, and e.g. mass. One of those variables, the parameter $\gamma$, describes how much space is curved by unit mass and equals unity in Einstein's theory. Expressed in the PPN formalism, the angle $\theta$ through which a radiowave is deflected is given approximately by (e.g. Shapiro et al., 2004)

$$
\theta \approx \frac{(1+\gamma) G M}{c^{2} b}(1+\cos \phi)
$$

where $G M$ denotes the product of the Newtonian gravitational constant and the mass of the considered gravitating body, $c$ the vacuum speed of light, $b$ the distance of closest approach of the radiowave's path to the center of the gravitating body, and $\phi$ is the angle between the gravitating body and the radio source as viewed from Earth. 
Besides the bending effect, interferometry is capable of measuring the temporal delay of radiowaves, also known as Shapiro delay (Fig. 1). In the case of VLBI the gravitational time delay is considered as

$$
\tau_{\mathrm{grav}}=(1+\gamma) \cdot \frac{G M}{c^{3}} \cdot \ln \left[\frac{\left|\mathbf{x}_{1}\right|+\mathbf{x}_{1} \cdot \mathbf{k}}{\left|\mathbf{x}_{2}\right|+\mathbf{x}_{2} \cdot \mathbf{k}}\right]
$$

where $\mathbf{x}_{\mathbf{i}}$ denotes the position vector of the i-th antenna with respect to the center of the gravitating body and $\mathbf{k}$ is the unit vector towards the radiosource as viewed from the Earth-bound baseline.

The partial derivative of the delay with respect to $\gamma$ is easily comprehensible:

$$
\frac{\partial \tau}{\partial \gamma}=\frac{G M}{c^{3}} \cdot \ln \left[\frac{\left|\mathbf{x}_{1}\right|+\mathbf{x}_{1} \cdot \mathbf{k}}{\left|\mathbf{x}_{2}\right|+\mathbf{x}_{2} \cdot \mathbf{k}}\right]
$$

Up to now several groups have determined the parameter $\gamma$ using the geodetic VLBI observations, which are provided by the International VLBI Service for Geodesy and Astrometry (IVS) (Tab. 1). All of those tests focus on the effects imposed by the Sun. However, there were also several efforts to observe Jupiter's deflection. Treuhaft \& Lowe (1991) tried to find the deflection by Jupiter experimentally using a single long baseline DSN experiment during a near-occultation event, which was proposed by Schuh et al. (1988). A comparable near-occultation happened in 2002 and was investigated by several groups, e.g. by Fomalont \& Kopeikin (2003).

In about mid 2002 a more stringent cut-off Sun elongation angle of about 14 deg was used for the IVS scheduling, significantly lowering the sensitivity of the VLBI observables during high solar activity, but unfortunately also for the gravitational time delay determination. Even with the new cut-off elongation angle geodetic VLBI remains competitive to $\gamma$-determinations by spacecraft ranging (Shapiro et al., 1971; Reasenberg et al., 1979; Bertotti et al., 2003), in particular due to the large amount of involved data observing at many epochs and in most directions of the universe. Without commenting on the reported formal errors of the spacecraft ranging analyses, it can be stated, that geodetic VLBI tends to handle commited and ommited errors rather conservatively. Thus, relativity remains a point of interest to the IVS and its Observing Program Committee (OPC) is open for suggestions to carry out special Research and Development (R\&D) observing sessions during relativistic scenarios.

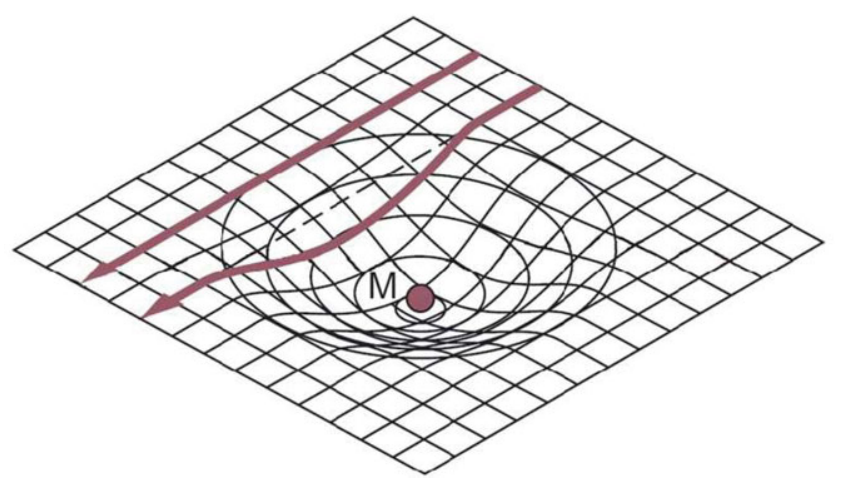

Figure 1. Gravitational time delay of radiowaves; 'Shapiro delay'. 
Table 1. Review of $\gamma$-determination using geodetic VLBI data.

\begin{tabular}{l|c|c|c|l}
\hline Author(s) & Year & $\gamma$ & $\sigma$ & Data \\
\hline Counselman et al. & 1974 & 0.98 & \pm 0.06 & 1972 occultation of 3C279 by the Sun \\
Fomalont \& Sramek & 1975 & 1.0075 & \pm 0.022 & 1974 occultation of 3C279 by the Sun \\
Fomalont \& Sramek & 1976 & 1.0035 & \pm 0.018 & 1974 and 1975 occultation of 3C279 by the Sun \\
Robertson \& Carter & 1984 & 1.008 & \pm 0.005 & MERIT, POLARIS, IRIS \\
Carter, Robertson \& MacKay & 1985 & 1.000 & \pm 0.003 & POLARIS, IRIS since 1980 \\
Robertson, Carter \& Dillinger & 1991 & 1.000 & \pm 0.002 & POLARIS, IRIS, CDP since 1980 \\
Lebach et al. & 1995 & 0.9996 & \pm 0.0017 & 1987 occultation of 3C279 by the Sun \\
Eubanks et al. & 1997 & 0.99994 & \pm 0.00031 & Geodetic VLBI sessions from 1979 to 1997 \\
Shapiro et al. & 2004 & 0.9998 & $\pm 0.0002^{1}$ & Geodetic VLBI sessions from 1979 to 1999 \\
Lambert \& Le Poncin-Lafitte & 2009 & 0.99984 & $\pm 0.00015^{2}$ & Geodetic VLBI sessions from 1979 to 2008 \\
\hline
\end{tabular}

Notes:

${ }^{1}$ Shapiro et al. (2004) estimate the standard error to 0.0004 , however their reported formal error is 0.0002 , which is used here to achieve a consistent comparison.

${ }^{2}$ Lambert \& Le Poncin-Lafitte (2009) claim an estimated limit of 0.0002 to the standard error.

\section{VLBI2010 and the relativistic delay model}

To fulfill the requirements of space geodesy, in particular specified through GGOS, the Global Geodetic Observing System of the IAG, and to overcome the current limitations of the existing VLBI infrastructure, the IVS, in 2003, established Working Group 3 (WG3) named VLBI2010. The goal of VLBI2010 is to significantly modernize the existing VLBI system towards

- a positional acuracy of $1 \mathrm{~mm}$,

- a velocity accuracy of $0.1 \mathrm{~mm} \cdot \mathrm{yr}^{-1}$,

- continuous monitoring of the Earth orientation parameters (EOP), and

- near real-time availibility of the results.

Working Group 3 closed its efforts presenting a Final Report to the IVS in 2005 (Niell et al., 2005). The progress towards a new VLBI system, however, was taken over by the VLBI2010 Committee (V2C), which was established to continue and realize the studies recommended by WG3. To move to these targets, V2C proposed a number of strategies including VLBI antenna system considerations (Tab. 2) and extensive simulation studies were carried out (Petrachenko et al., 2009).

Table 2. VLBI2010: System characteristics.

\begin{tabular}{|c|c|c|}
\hline & Current VLBI & VLBI2010 \\
\hline Antenna size & 5 to $100 \mathrm{~m} \mathrm{dish}$ & about $12 \mathrm{~m}$ dish \\
\hline Slew speed & about 20 to $200 \mathrm{deg} \cdot \mathrm{min}^{-1}$ & $\geqslant 360 \mathrm{deg} \cdot \min ^{-1}$ \\
\hline Sensitivity & 200 to $15,000 \mathrm{SEFD}$ & $\leqslant 2,500 \mathrm{SEFD}$ \\
\hline Frequency range & $\mathrm{S} / \mathrm{X}$ band & about 2 to $15 \mathrm{GHz}$ \\
\hline Recording rate & 128 or $256 \mathrm{Mbps}$ & 8 to $16 \mathrm{Gbps}$ \\
\hline Data transfer & $\begin{array}{l}\text { usually ship disks, } \\
\text { some e-transfer }\end{array}$ & $\begin{array}{c}\text { e-transfer, e-VLBI } \\
\text { ship disks when required }\end{array}$ \\
\hline
\end{tabular}


The current conventional relativistic VLBI model (IERS, 2004, chapter 11) is based on the 'consensus model' (Eubanks, 1991), which united various VLBI delay models prior to 1991. Klioner (1991) presented an additional model, which considers sources at finite distances, e.g. artificial satellites or spacecrafts and baselines including space-borne or orbiting telescopes as well. At that time a precision of $1 \mathrm{ps}$ was aimed for and remained sufficient until now. For the target accuracy of VLBI2010, however, it will be necessary to revisit the consensus model and to assess, whether it includes all terms down to the order of $0.3 \mathrm{ps}$.

\section{Conclusion and outlook}

Astrometric and geodetic VLBI is able to measure effects of general relativity, the relativistic deflection and the relativistic time delay (Shapiro delay). With its huge amount of observations in all directions of the universe, currently about 5 million delays over about 30 years, the IVS maintains universal, reliable, and robust data for applied relativity. Simulations have shown, that a network of VLBI2010 stations provides the same amount of data ( 5 million observations) in a few weeks. Future developments in view of VLBI2010 foresee significant improvements and demand the relativistic VLBI delay model to hold for a slightly better $(0.3 \mathrm{ps})$ accuracy. Besides the Sun and the Earth, the gravitational time delays of Jupiter, Saturn, Venus, and the Moon will have to be considered in standard VLBI2010 analyses.

The main factors of uncertainty of VLBI are

- the intrinsic source structure,

- the wet neutral atmosphere contribution,

- the uneven North-South distribution of the network, and

- the solar coronal plasma for smaller Sun elongations.

With structure corrections and special VLBI sessions dedicated to general relativity the accuracy of the $\gamma$-determination could be significantly better than $10^{-4}$.

\section{References}

Bertotti, B., Iess, L., \& Tortora, P. 2003, A test of general relativity using radio links with the Cassini spacecraft. Nature, 425, 374-376

Carter, W. E., Robertson, D. S., \& MacKay, J. R. 1985, Geodetic Radio Interferometric Surveying: Applications and Results. Journal of Geophysical Research, 90, B6, 4577-4587

Counselman, C. C., Kent, S. M., Knight, C. A., Shapiro, I. I., Clark, T. A., Hinteregger, H. F., Rogers, A. E. E., \& Whitney, A. R. 1974, Solar Gravitational Deflection of Radio Waves Measured by Very-Long-Baseline Interferometry. Physical Review Letters, 33, 27, 1621-1623

Einstein, A. 1908, Über das Relativitätsprinzip und die aus demselben gezogenen Folgerungen. Jahrbuch der Radioaktivität und Elektronik, IV., 4., 411-462

Einstein, A. 1911, Über den Einfluß der Schwerkraft auf die Ausbreitung des Lichtes. Annalen der Physik, 35, 898-908

Eubanks, T. M. 1991, A Consensus Model for Relativistic Effects in Geodetic VLBI. In: Eubanks, T. M. (ed.), Proceedings of the USNO Workshop on Relativistic Models for use in Space Geodesy, 60-82

Eubanks, T. M., Martin, J. O., Archinal, B. A., Josties, F. J., Klioner, S. A., Shapiro, S., \& Shapiro, I. I. 1997, Advances in Solar System Tests of Gravity. American Physical Society, APS/AAPT Joint Meeting, April 18-21, 1997, abstract \#K11.05 
Fomalont, E. B. \& Sramek, R. A. 1975, A confirmation of Einstein's general theory of relativity by measuring the bending of microwave radiation in the gravitational field of the Sun. The Astronomical Journal, 199, 3, 749-755

Fomalont, E. B. \& Sramek, R. A. 1976, Measurement of the Solar Gravitational Deflection of Radio Waves in Agreement with General Relativity. Physical Review Letters, 36, 25, $1475-1478$

Fomalont, E. B. \& Kopeikin, S. M. 2003, The Measurement of the Light Deflection from Jupiter: Experimental Results. arXiv:astro-ph/0302294v2, 1-10

IERS: IERS Conventions (2003). 2004, in: McCarthy D. D. \& Petit, G. (eds.) IERS Technical Note, 32

Klioner, S. A. 1991, General relativistic model of VLBI observables. In: U. S. Department of Commerce. National Oceanic and Atmospheric Administration. National Ocean Service (eds.), Proceedings of the AGU Chapman Conference on Geodetic VLBI: Monitoring Global Change, NOAA Technical Report NOS 137 NGS 49, 188-202

Lambert, S. B. \& Le Poncin-Lafitte, C. 2009, Determination of the relativistic parameter $\gamma$ using very long baseline interferometry. Astronomy \& Astrophysics, arXiv:0903.1615v1, 1-6

Lebach, D. E., Corey, B. E., Shapiro, I. I., Ratner, M. I., Webber, J. C., Rogers, A. E. E., Davis, J. L., \& Herring, T. A. 1995, Measurement of the Solar Gravitational Deflection of Radio Waves Using Very-Long-Baseline Interferometry. Physical Review Letters, 75, 8, 1439-1442

Niell, A. et al. 2005, VLBI2010: Current and future requirements for Geodetic VLBI Systems. http://ivscc.gsfc.nasa.gov/about/wg/wg3/IVS_WG3_report_050916.pdf, 32

Petrachenko, B. et al. 2009, Design Aspects of the VLBI2010 System. Progress Report of the IVS VLBI2010 Committee. In: Behrend D. \& K. Baver (eds.), 2008 IVS Annual Report, ftp://ivscc.gsfc.nasa.gov/pub/misc/V2C/PR-V2C_090417.pdf, 56

Reasenberg, R. D. et al. 1979, VIKING relativity experiment: verification of signal retardation by solar gravity. The Astronomical Journal, 234, L219-L221

Robertson, D. S. \& Carter, W. E. 1984, Relativistic deflection of radio signals in the solar gravitational field measured with VLBI. Nature 310, 572-574

Robertson, D. S., Carter, W. E., \& Dillinger, W. H. 1991, New measurement of the solar gravitational deflection of radio signals using VLBI. Nature, 349, 768-770

Schuh, H., Fellbaum, M., Campbell, J., Soffel, M., Ruder, H., \& Schneider, M. 1988, On the deflection of radio signals in the gravitational field of Jupiter. Physics Letters A, 129, 5-6, $299-300$

Seielstad, G. A., Sramek, R. A., \& Weiler, K. W. 1970, Measurement of the deflection of 9.602GHz radiation from 3C279 in the solar gravitational field. Physical Review Letters, 24, 24, 1373-1376

Shapiro, I. I. 1967, New Method for the Detection of Light Deflection by Solar Gravity. Science, $157,806-808$

Shapiro, I. I. et al. 1971, Fourth Test of General Relativity: New Radar Result. Physical Review Letters, 26, 18, 1132-1135

Shapiro, S. S., Davis, J. L., Lebach, D. E., \& Gregory, J. S. 2004, Measurement of the Solar Gravitational Deflection of Radio Waves using Geodetic Very-Long-Baseline Interferometry Data, 1979-1999. Physical Review Letters, 92, 12, 121101 1-4

Soffel, M. H. 1989, Relativity in Astrometry, Celestial Mechanics and Geodesy. Springer, Berlin, Heidelberg, New York, A\&A library, ISBN 3-540-18906-8, 208

Texas Mauritanian Eclipse Team (Brune, R. A. Jr., Cobb, C. L., DeWitt, B. S. et al.) 1976, Gravitational deflection of light: solar eclipse of 30 June 1973 I. Description of procedures and final results. The Astronomical Journal, 81, 6, 452-454

Treuhaft, R. N. \& Lowe, S. T. 1991, A measurement of planetary relativistic deflection. The Astronomical Journal, 102, 5, 1879-1888 